\title{
Survival outcomes of neoadjuvant versus adjuvant chemotherapy in triple-negative breast cancer: a meta-analysis of 36,480
} cases

\author{
Lin-Yu Xia ${ }^{1 *}$ (D), Qing-Lin Hü, Jing Zhang ${ }^{2}$, Wei-Yun Xu ${ }^{2}$ and Xiao-Shi Li ${ }^{1}$
}

\begin{abstract}
Background: The survival outcomes of neoadjuvant chemotherapy (NACT) versus adjuvant chemotherapy (ACT) for patients with triple-negative breast cancer (TNBC) remain unclear. Therefore, in this study, a meta-analysis was conducted to analyze current evidence on the survival outcomes of NACT versus ACT in TNBC.

Methods: A systematic search was performed on the PubMed and Embase databases to identify relevant articles investigating the survival outcomes of NACT versus ACT in TNBC.

Results: A total of nine studies involving 36,480 patients met the selection criteria. Among them, 10,728 (29.41\%) received NACT, and 25,752 (70.59\%) received ACT. The pathological complete response (pCR) rate was $35 \%(95 \% \mathrm{Cl}$ $=0.23-0.48$ ). Compared with $A C T$, the overall survival (OS) of NACT was poor ( $H R=1.59 ; 95 \% \mathrm{Cl}=1.25-2.02 ; P=$ $0.0001)$, and there was no significant difference in disease-free survival (DFS) between the two treatments ( $H R=$ $0.85 ; 95 \% \mathrm{Cl}=0.54-1.34 ; P=0.49)$. NACT with $\mathrm{pCR}$ significantly improved the OS (HR $=0.53 ; 95 \% \mathrm{Cl}=0.29-0.98 ; P$ $=0.04)$ and DFS $(H R=0.52 ; 95 \% \mathrm{Cl}=0.29-0.94 ; P=0.03)$, while the OS $(H R=1.18 ; 95 \% \mathrm{Cl}=1.09-1.28 ; P<0.0001)$ and DFS ( $\mathrm{HR}=2.36 ; 95 \% \mathrm{Cl}=1.42-3.89 ; P=0.0008)$ of patients with residual disease (RD) following NACT were worse compared to those receiving ACT.
\end{abstract}

Conclusion: These findings suggest that, for TNBC, NACT with pCR is superior to ACT in improving OS and DFS, and it turns to be opposite when patients are receiving NACT with RD.

Keywords: Triple-negative breast cancer, Neoadjuvant, Adjuvant chemotherapy, Survival outcomes, Meta-analysis

\section{Introduction}

Breast cancer is the most common cancer in women. Globally, nearly 1.2 million to 1.4 million women are diagnosed with breast cancer, and about 400,000 die of breast cancer [1]. TNBC is defined as a type of breast cancer lacking the three most common types of

\footnotetext{
* Correspondence: lylc1023@163.com

'Department of Thyroid and Breast Surgery, The First Affiliated Hospital of Chengdu Medical College, 278 Baoguang Avenue Middle Section, Xindu District, Chengdu City 610500, Sichuan Province, China

Full list of author information is available at the end of the article
}

receptors namely, estrogen receptor (ER), progesterone receptor (PR), and HER-2 expression which are known to drive the growth of breast cancer. TNBC accounts for $12-20 \%$ of all breast cancers, and it is characterized by high pathological grade, strong invasiveness, local recurrence, high metastasis rate, and poor prognosis [2-4]. Therefore, systemic treatment should be administered in the early stage after diagnosis. In the past, ACT has been the standard treatment for TNBC, but now more and more patients with TNBC have adopted NACT because it can control systemic micrometastases, reduce the

C C The Author(s). 2020 Open Access This article is licensed under a Creative Commons Attribution 4.0 International License, which permits use, sharing, adaptation, distribution and reproduction in any medium or format, as long as you give appropriate credit to the original author(s) and the source, provide a link to the Creative Commons licence, and indicate if changes were made. The images or other third party material in this article are included in the article's Creative Commons licence, unless indicated otherwise in a credit line to the material. If material is not included in the article's Creative Commons licence and your intended use is not permitted by statutory regulation or exceeds the permitted use, you will need to obtain permission directly from the copyright holder. To view a copy of this licence, visit http://creativecommons.org/licenses/by/4.0/. The Creative Commons Public Domain Dedication waiver (http://creativecommons.org/publicdomain/zero/1.0/) applies to the data made available in this article, unless otherwise stated in a credit line to the data. 
tumor burden, provide surgical or conservative breast surgery opportunities for locally advanced breast cancer patients, and allow detection of tumor sensitivity to chemotherapeutic drugs. pCR after NACT improves tumorfree survival rate of patients [5, 6]. Studies have shown that the rate of $\mathrm{pCR}$ in patients with $\mathrm{TNBC}$ receiving NACT is significantly higher than that of non-TNBC patients [7-11]. This indicates that NACT is effective for TNBC patients. Currently, studies have compared the prognosis of NACT and ACT in patients with TNBC, but the results are contradictory [12-14]. Thus, whether NACT yields better survival outcomes in TNBC than ACT is still controversial. Our study aimed to compare the survival outcomes of NACT versus ACT in TNBC by meta-analysis.

\section{Material and methods}

\section{Search strategy}

A systematic literature search was performed on the PubMed and Embase databases for the period up to January 18, 2020, to identify eligible studies. The keywords used in the search strategy were triple-negative breast neoplasms OR triple-negative breast cancer OR triple-negative breast carcinoma AND neoadjuvant OR preoperative AND Adjuvant chemotherapy OR chemotherapy. A total of nine articles with a total of 36,480 patients met the eligibility criteria [12-20]. The inclusion of studies was not limited to geographical location of study or publication language.

\section{Inclusion and exclusion criteria}

Eligible studies met the following inclusion criteria to ensure only high-quality studies were considered for this analysis. Inclusion criteria are as follows: (1) patients diagnosed with TNBC, (2) the study compared the survival outcomes of NACT with ACT, (3) the study assessed the overall prognosis of TNBC, (4) the study reported survival outcomes in terms of OS and/or DFS. The exclusion criteria were as follows: (1) articles lacking the original data, (2) studies lacking information on survival outcomes in TNBC, (3) articles not reporting or giving an estimate of the hazard ratio (HR) and a 95\% confidence interval $(95 \% \mathrm{CI})$. Figure 1 illustrates the eligibility criteria of articles enrolled in this meta-analysis.

\section{Data extraction and quality assessment}

A standardized data extraction form was used to extract relevant information from each study. For each eligible study, the following information was extracted: the first author's name, publication year, patients' countries, patients' ages, study design, number of participations, tumor characteristics, chemotherapy regimen, and follow-up results.
The Newcastle-Ottawa quality assessment scale was used to assess the quality of each included study [21]. The NOS evaluated non-randomized studies based on three criteria: patient selection (four stars), study group comparability (two stars), and outcome evaluation (three stars). Only studies with a score of 6 or above were included in the meta-analysis (See Supplementary Table 1, Additional File 1).

\section{Statistical analysis}

Meta-analysis was conducted using RevMan version 5.3 (RevMan, version 5.3 for Windows; Cochrane Collaboration, Oxford, UK). The hazard ratios (HRs) with 95\% CIs were calculated to estimate the association between the DFS and OS of NACT and ACT in TNBC. Published data and Kaplan-Meier survival curves were used to extract the HR estimates according to the methods reported by Tierney et al. if the HRs were not directly provided [22]. Chi-squared based Q-test $(P>0.10)$ and $I^{2}$ were used to determine statistical heterogeneity within the studies. When $I^{2}<50 \%$, the studies were considered to have acceptable heterogeneity, and the fixed-effects model was used. Otherwise, the random-effects model was used. All $P$ values were two-sided, and $P<0.05$ was considered to be statistically significant. Publication bias was assessed using funnel's plot and quantified by Egger's test [23]. These analyses were performed using Stata 15.0 (StataCorp, College Station, Tex).

\section{Results}

Figure 1 shows the process of selecting the included studies. A total of 420 articles were first identified for evaluation. Among these, 158 were excluded because they were duplicates while 238 were irrelevant to this study. Based on the inclusion and exclusion criteria described above, 15 were excluded from further analysis. Therefore, 9 publications were eligible for the metaanalysis. Among the 9 studies, 7 were retrospective while 2 were prospective. Tables 1 and 2 present the characteristics of the included studies.

Among the 36,480 patients included, 10,728 (29.41\%) received NACT, and 25,752 (70.59\%) received ACT. A pCR rate of $35 \%(95 \% \mathrm{CI}=0.23-0.48)$ was obtained in 6172 patients receiving NACT from six studies [12, 13, 17-20] (Fig. 2), and heterogeneity was detected in these data $\left(I^{2}=96 \%, P<0.01\right)$. Three studies reported the breast conserving surgery rate, from which we found that the breast conserving rate after NACT was lower than ACT $(\mathrm{RR}=0.84 ; 95 \% \mathrm{CI}=0.57-1.23 ; P=0.37)$ [12, 14, 20] (Fig. 3), and heterogeneity was detected in these data $\left(I^{2}=82 \%, P=0.004\right)$.

The OS of NACT versus ACT in TNBC in the entire study was evaluated in the Clifton, Fisher, Kennedy, Cheng, Yang, Biswas, Bagegni, and Philipovskiy trials 


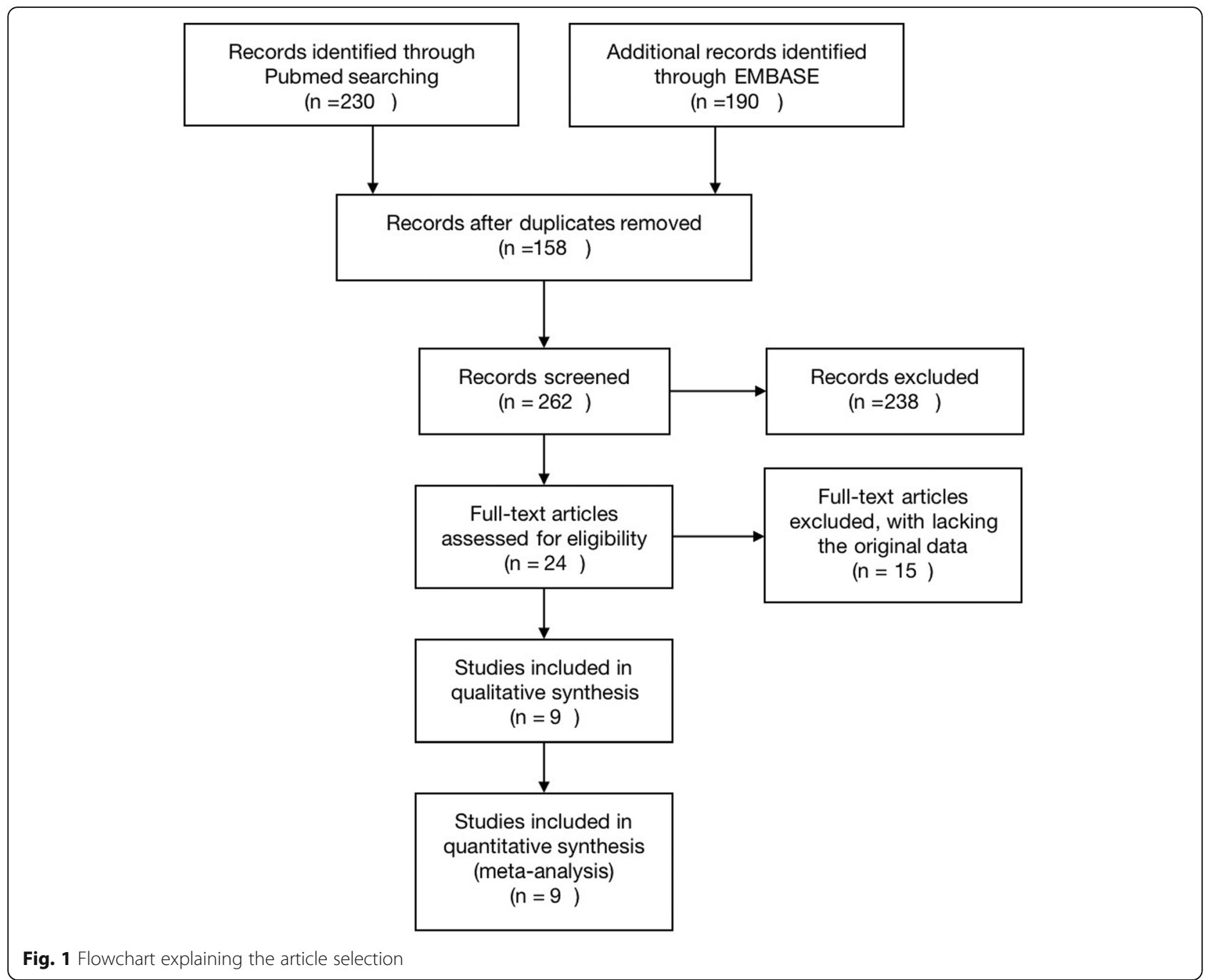

with a sample size of 36,422 [12-14, 16-20]. After a median follow-up of 4.12 years, NACT led to worse OS than ACT with an HR of 1.59; 95\% CI (1.25-2.02); $P=$ 0.0001 . Significant heterogeneity existed among the studies $\left(I^{2}=88 \%, P<0.000001\right)$. Unlike TNBC patients who received $\mathrm{ACT}$, those with $\mathrm{RD}$ who were put on NACT had worse OS $(\mathrm{HR}=1.18 ; 95 \% \mathrm{CI}=1.09-1.28 ; P$ $<0.0001$ ), while those who achieved pCR following NACT had significant OS benefits $(\mathrm{HR}=0.53 ; 95 \% \mathrm{CI}=$ 0.29-0.98; $P=0.04$ ) in the studies of Clifton, Fisher,

Table 1 Main characteristics and results of the eligible studies

\begin{tabular}{|c|c|c|c|c|c|c|c|c|c|c|}
\hline First author & Year & Country & Study type & N & Clinical stage & Chemotherapy regimen & pCR rate & $\begin{array}{l}\text { Follow-up } \\
\text { (median) (year) }\end{array}$ & HR estimation & Outcomes \\
\hline Clifton & 2018 & USA & Prospective & 319 & $|-| \mid$ & $\mathrm{A} / \mathrm{T}$ & 0.538 & 6.33 & Survival curve & OS, DFS \\
\hline Fisher & 2012 & USA & Retrospective & 385 & $|-|||$ & NA & 0.17 & 2.5 & Survival curve & OS \\
\hline Kennedy & 2010 & USA & Retrospective & 405 & $|-|||$ & NA & NR & 4.3 & Survival curve & OS \\
\hline Sharma & 2015 & USA & Retrospective & 146 & $|-| \mid$ & $\mathrm{A} / \mathrm{T}$ & NR & 3.08 & Given by author & DFS \\
\hline Cheng & 2017 & NR & Retrospective & 15,483 & $|-|||$ & NA & NR & 2 & Given by author & OS \\
\hline Yang & 2018 & China & Prospective & 67 & $\|-1\|$ & $\mathrm{A} / \mathrm{T}$ & 0.194 & 6.5 & Given by author & OS \\
\hline Biswas & 2017 & USA & Retrospective & 420 & $|-|||$ & $\mathrm{A} / \mathrm{T}$ & 0.33 & 3.9 & Survival curve & OS \\
\hline Bagegni & 2019 & USA & Retrospective & 19,151 & $\|-\| \|$ & NA & 0.474 & 2.5 & Survival curve & OS \\
\hline Philipovskiy & 2019 & USA & Retrospective & 104 & $|-|||$ & $\mathrm{A} / \mathrm{T}$ & 0.4 & 6 & Given by author & OS,DFS \\
\hline
\end{tabular}


Table 2 Patient and tumor characteristics in the neoadjuvant and adjuvant groups from the studies

\begin{tabular}{|c|c|c|c|c|c|c|c|c|c|c|}
\hline \multirow[t]{2}{*}{ First author } & \multirow{2}{*}{$\begin{array}{l}\text { N NACT/ } \\
\text { ACT }\end{array}$} & \multirow{2}{*}{$\begin{array}{l}\text { Median age } \\
\text { (year) } \\
\text { NACT/ACT }\end{array}$} & \multicolumn{2}{|l|}{ Clinical stage } & \multicolumn{2}{|c|}{ Nuclear grade } & \multicolumn{2}{|l|}{ Histology } & \multicolumn{2}{|c|}{$\begin{array}{l}\text { Local } \\
\text { treatment }\end{array}$} \\
\hline & & & $\overline{\mathrm{NACT}}$ & ACT & $\overline{\mathrm{NACT}}$ & $\mathrm{ACT}$ & $\overline{\mathrm{NACT}}$ & $\mathrm{ACT}$ & & \\
\hline & & & $|/||/|||$ & $|/||| /|| \mid$ & $1 / 2 / 3$ & $1 / 2 / 3$ & $\begin{array}{l}\text { IDC/ILC/ } \\
\text { Other }\end{array}$ & $\begin{array}{l}\text { IDC/ILC/ } \\
\text { Other }\end{array}$ & $M$ & $B$ \\
\hline \multirow[t]{2}{*}{ Clifton } & $132 / 187$ & $<50,102 / 144$ & 15/70/0 & $20 / 65 / 0$ & $0 / 4 / 81$ & $2 / 1 / 79$ & $84 / 0 / 1$ & $83 / 0 / 2$ & 162 & 157 \\
\hline & & $\geq 50,30 / 43$ & & & & & & & & \\
\hline \multirow[t]{2}{*}{ Fisher } & $151 / 234$ & $<50,82 / 96$ & $10 / 85 / 49$ & $81 / 91 / 11$ & $2 / 15 / 130$ & $2 / 25 / 200$ & $120 / 7 / 24$ & 190/7/37 & NR & NR \\
\hline & & $\geq 50,69 / 138$ & & & & & & & & \\
\hline Kennedy & $154 / 251$ & $50 / 53$ & $3 / 80 / 43$ & 89/101/19 & $0 / 14 / 135$ & 5/34/197 & $130 / 14 / 10$ & $198 / 28 / 25$ & 207 & 198 \\
\hline Sharma & $67 / 79$ & $52 / 58$ & NR & $N R$ & NR & NR & NR & $N R$ & NR & NR \\
\hline \multirow[t]{2}{*}{ Cheng } & $\begin{array}{l}4335 / 11 \\
148\end{array}$ & $<50,1951 / 3456$ & NR/NR/1517 & NR/NR/669 & NA & NA & NR & $N R$ & NR & NR \\
\hline & & $\geq 50,2384 / 7692$ & & & & & & & & \\
\hline Yang & $36 / 31$ & NA & NA & NA & NR & $N R$ & NA & NA & NR & NR \\
\hline Biswas & $202 / 218$ & $51 / 51$ & $1 / 105 / 96$ & $69 / 117 / 32$ & NA & NA & $N R$ & $N R$ & NA & NA \\
\hline Bagegni & $\begin{array}{l}5621 / 13 \\
530\end{array}$ & $51.9 / 55.7$ & $\begin{array}{l}0 / 3843 / \\
1778\end{array}$ & $\begin{array}{l}0 / 12142 / 13 \\
88\end{array}$ & $\begin{array}{l}26 / 649 / \\
4530\end{array}$ & $\begin{array}{l}\text { 102/1328/11, } \\
391\end{array}$ & $N R$ & $N R$ & NR & NR \\
\hline Philipovskiy & $30 / 74$ & $50.4 / 53$ & $3 / 11 / 16$ & $16 / 41 / 17$ & NR & NR & & $0 / 4^{\mathrm{a}}$ & 45 & 54 \\
\hline
\end{tabular}

IDC invasive ductal carcinoma, ILC invasive lobular carcinoma, NR not reported, NA not replied, $M$ mastectomy, $B$ breast conserving surgery

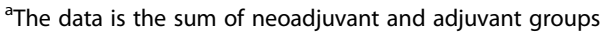

Bagegni, and Philipovskiy [12, 13, 19, 20]. Heterogeneity did not exist among the included studies as shown in Fig. 4.

The trials by Clifton, Sharma, and Philipovskiy [12, 15, 20] reported DFS after NACT versus ACT in TNBC. A total of 569 patients were included in the pooled analysis with a median follow-up time of 5.14 years. There was no statistically significant difference between NACT and ACT arms on the DFS (HR $=0.85 ; 95 \%$ CI $=0.54-1.34$; $P=0.49)$. Compared with patients receiving ACT, those who underwent NACT with pCR had a better DFS (HR $=0.52 ; 95 \% \mathrm{CI}=0.29-0.94 ; P=0.03)$, and those with RD had a worse DFS $(\mathrm{HR}=2.36 ; 95 \% \mathrm{CI}=1.42-3.89 ; P$
$=0.0008)$. Heterogeneity did not exist among the included studies as shown in Fig. 5.

Publication bias was detected by funnel plots and Egger's test (See Supplementary Figure 1, Additional File 2). All $P$ values were $>0.05$ (See Supplementary Table 2, Additional File 3$)$, indicating no potential publication bias.

\section{Discussion}

TNBC is an aggressive form of breast cancer which is highly invasive and prone to recurrence and metastasis. We analyzed 36,480 patients in 9 studies and concluded that for TNBC patients, the OS of ACT exceeds that of NACT but there was no significant difference in DFS

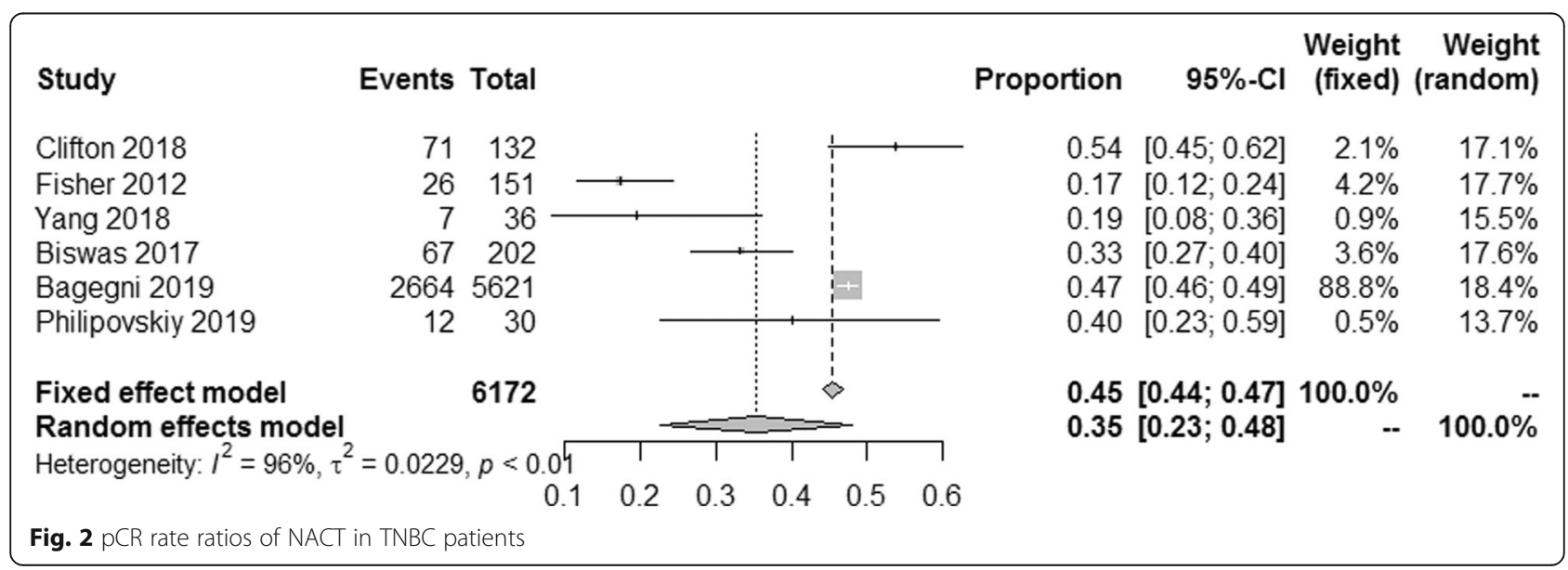




\begin{tabular}{|c|c|c|c|c|c|c|c|c|c|c|}
\hline Studv or Subgroup & \multicolumn{2}{|c|}{ neoadjıvant } & \multicolumn{2}{|c|}{ adjıvant } & Weight & $\begin{array}{c}\text { Risk Ratio } \\
\text { M-H, Random, } 95 \% \mathrm{Cl}\end{array}$ & \multicolumn{4}{|c|}{$\begin{array}{c}\text { Risk Ratio } \\
\text { M-H, Random, } 95 \% \mathrm{Cl}\end{array}$} \\
\hline Clifton 2018 & 48 & 132 & 109 & 187 & $35.3 \%$ & $0.62[0.48,0.81]$ & & $=$ & & \\
\hline Kennedy 2010 & 79 & 154 & 119 & 251 & $37.6 \%$ & $1.08[0.88,1.32]$ & & & & \\
\hline Philipovskiy 2019 & 14 & 30 & 40 & 74 & $27.1 \%$ & $0.86[0.56,1.34]$ & & & & \\
\hline Total $(95 \% \mathrm{Cl})$ & & 316 & & 512 & $100.0 \%$ & $0.84[0.57,1.23]$ & & & & \\
\hline Total events & 141 & & 268 & & & & & & & \\
\hline $\begin{array}{l}\text { Heterogeneity: Tauz } \\
\text { Test for owerall effect }\end{array}$ & $\begin{array}{l}0.09 ; \mathrm{Chi} \\
Z=0.91\end{array}$ & $\begin{array}{l}=11.0 \\
=0.37\end{array}$ & $6, d f=2($ & $P=0.0$ & $104) ;\left.\right|^{2}=8$ & $2 \%$ & 0.01 & $\begin{array}{c}0.1 \\
\text { Favours [NACT] }\end{array}$ & $\begin{array}{c}10 \\
\text { Favours [ACT] }\end{array}$ & 100 \\
\hline
\end{tabular}

between the two treatments. Compared with ACT, patients with RD following NACT had worse OS and DFS, while those who achieved pCR had a better OS and DFS.

Three large randomized trials estimating NACT and ACT, NSABP B-18, EORTC 10902, and IBBGS, found that there was no significant difference in survival between NACT and ACT [24-26]. However, these trials did not differentiate breast cancer molecular subtypes. The results of our study only apply to the TNBC subtype of breast cancer. In our analysis, ACT was superior to NACT in improving the survival outcomes. This may be related to the fact that patients with severe disease were

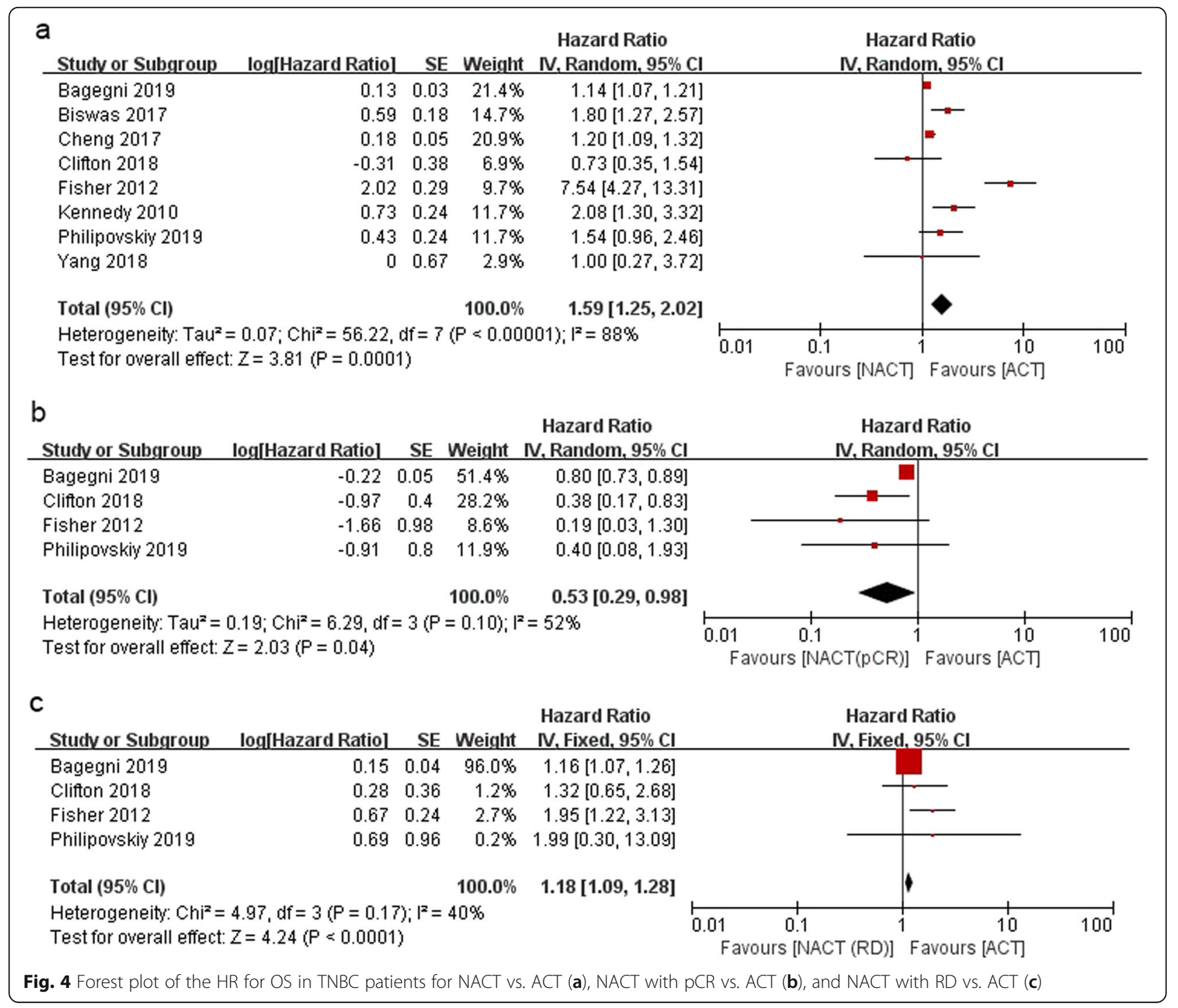




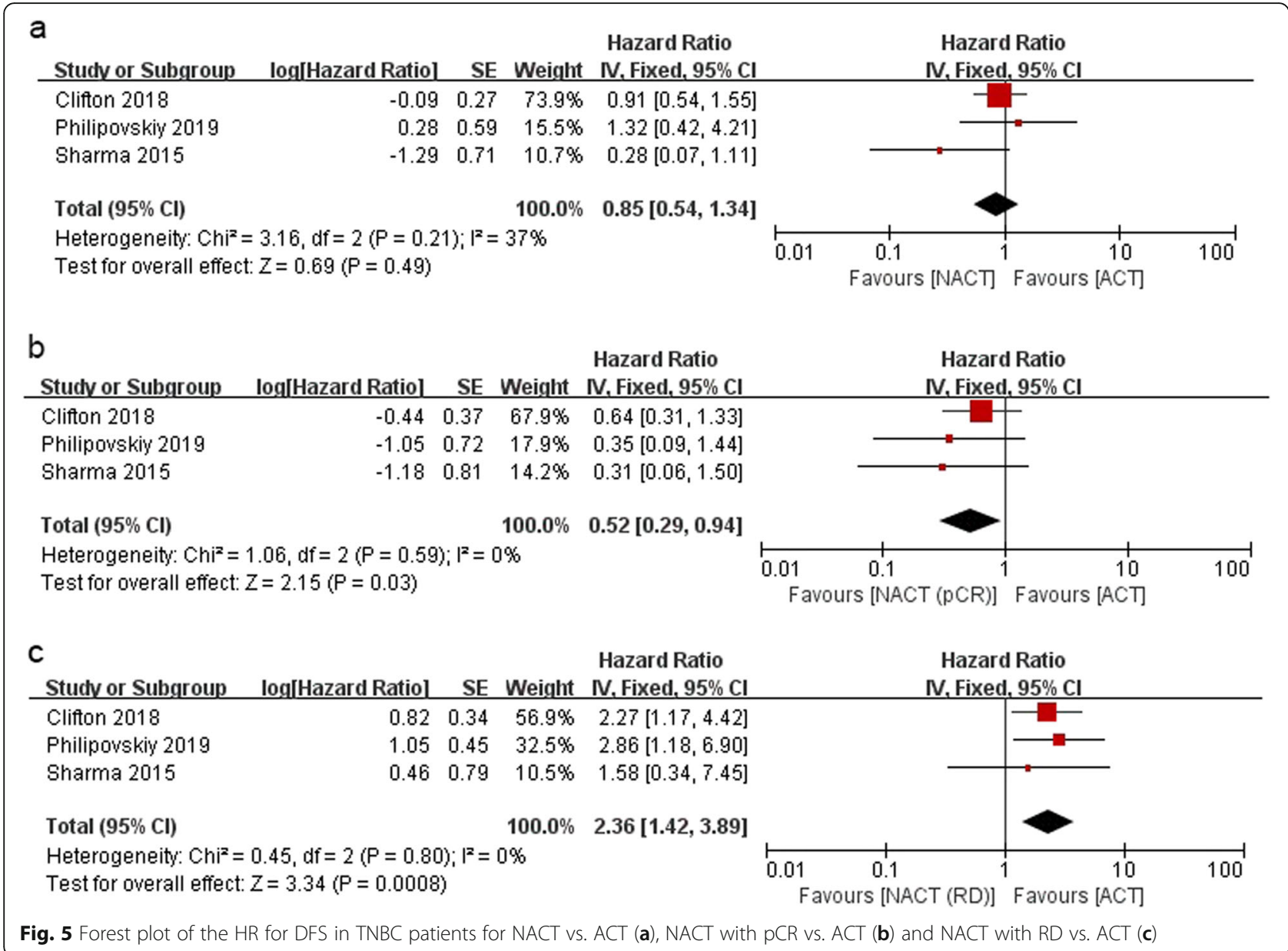

more likely to receive NACT. Studies have shown that patients receiving NACT have larger tumors, nodal positivity, and advanced clinical stage compared to those receiving ACT [13, 14, 16]. Although they try to control these factors that may affect the survival results in the multivariate model, we did not exclude some factors that are not included in the model which can potentially interfere with the choice of NACT or ACT. So the worse outcome of NACT may be partially caused by the biology of disease, so it is still noteworthy. Another reason for the lower OS in patients with TNBC who received NACT is the "delay effect" [14]. TNBC is characterized by increased risk of bone and central nervous system metastasis [27, 28]. NACT does not kill tumors when used for the first time as surgery does. Hence, patients receiving NACT may develop axillary metastases. Therefore, the observed survival benefit of ACT in our study may be a result of earlier tumor debulking, decreased opportunity for systemic tumor seeding, and systemic micro-metastases.

Consistent with our study, other studies have confirmed that patients with TNBC have a better prognosis after achieving pCR following NACT [7, 29]. Although patients who received NACT may have advanced disease, achieving pCR following NACT significantly improved survival. This suggests that in our study, compared with all patients receiving $\mathrm{NACT}$, the survival advantage of ACT is determined by the residual disease after NACT. In our meta-analysis, the pCR rate is $35 \%$ (95\% CI $=0.23-0.48 ; P<0.01)$ (Fig. 2). In these studies, patients with early stage, small tumor and negative lymph node were more likely to achieve $\mathrm{pCR}$. All studies reported a pCR rate below 50\%, except that of Clifton where the pCR rate was $54 \%$. Therefore, the high rate of $\mathrm{RD}$ is associated with a poor survival rate of NACT.

In this meta-analysis, the DFS was not significantly different between NACT and ACT arms. This differs from other studies. A study involving 4756 breast cancer patients showed that women who received NACT had higher local recurrence rates within 15 years $(21.4 \%$ vs 15.9\%) than those who received ACT (RR 1.37; 95\% CI $=1.17-1.61 ; P=0.0001$ ) [30]. In the 4756 breast cancer patients, the risk increased significantly after $0-4$ years (RR 1.35; 95\% CI $=1.11-1.64)$ and 5-9 years $(R R=1.53$; $95 \% \mathrm{CI}=1.08-2.17)$. Women who received NACT in their study were more likely to take breast-conserving 
treatments than those who received ACT (65\% vs $49 \%$ ). Breast-conserving surgery after NACT may increase the risk of local recurrence. Mauri et al. validated this through a meta-analysis of 9 randomized trials involving 3946 patients. They found that the risk of local recurrence of NACT group was significantly higher than that of ACT group due to the higher breast-conserving surgery rate in NACT cohort $(\mathrm{RR}=1.22 ; 95 \% \mathrm{CI}=1.04-$ $1.43 ; P=0.018$ ) [31]. Local recurrence following breastconserving surgery may be caused by the disunity of tumor regression model after NACT, the difficulty of locating tumors, and breast-conserving surgery [32, 33]. By contrast, there was no significant difference in breastconserving surgery rate between NACT and ACT $(\mathrm{RR}=$ 0.84; $95 \% \mathrm{CI}=0.57-1.23 ; P=0.37$ ) (Fig. 3 ) in this metaanalysis. This can be used to explain the discrepancies between our results and those reported in other studies.

This meta-analysis has some limitations. One of the limitations is that 8 studies analyzed the overall survival benefits of TNM stage I-III patients without distinguishing the early and late stages of the disease. Hence, we did not compare the survival benefits according to different stages of the disease. As proved in this study, TNBC with negative lymph node, small tumor, and early stage are more likely to achieve pCR in NACT. The poor survival benefit of NACT compared with ACT is determined by patients with RD. If all patients were in the early stage of disease, NACT may yield a higher pCR rate and a better survival benefit than ACT. Despite this limitation, we conclude that NACT with $\mathrm{pCR}$ can significantly improve survival in TNBC. In addition, the HR and $95 \% \mathrm{CI}$ extracted from the survival curves may be less reliable than those directly obtained from the articles. Finally, 7 studies included in our analysis were retrospective researches, and they probably have potential biases.

\section{Conclusion}

This meta-analysis shows that ACT results in a better OS for TNBC patients than NACT. However, there is no significant difference in DFS between the two treatments. Notably, NACT improves OS and DFS in patients achieving pCR. Thus, NACT may be more effective in patients predicted to achieve $\mathrm{pCR}$, while ACT is suitable for patients who cannot achieve pCR. We recommend that well-designed trials be conducted to confirm our results.

\section{Supplementary information}

Supplementary information accompanies this paper at https://doi.org/10. 1186/s12957-020-01907-7.

Additional file 1: Supplementary Table 1. Risk of bias in the included cohort studies (by the Newcastle-Ottawa quality assessment tool)
Additional file 2: Supplementary Figure 1. Funnel plot of the HR for OS in TNBC patients for NACT vs. ACT (a), NACT with pCR vs. ACT(b) NACT with RD vs. ACT(c), for DFS in TNBC patients for NACT vs. ACT (d), NACT with pCR vs. ACT(e) and NACT with RD vs. ACT(f)

Additional file 3: Supplementary Table 2. Results of Egger's tests for publication bias

\section{Abbreviations}

NACT: Neoadjuvant chemotherapy; ACT: Adjuvant chemotherapy;

TNBC: Triple-negative breast cancer; pCR: Pathological complete response;

OS: Overall survival; DFS: Disease-free survival; RD: Residual disease

\section{Acknowledgements}

Not applicable.

\section{Authors' contributions}

Protocol/project development: L.Y.X and Q.-L.H. Data acquisition and interpretation of data: L.Y.X, J.Z, W.-Y.X, and X.-S.L. Statistics analysis of data: L.-Y.X and J.Z. Manuscript drafting: L.-Y.X and Q.-L.H. Manuscript revision and accountable for all aspects of the study: L.-Y.X and J.Z. All authors read and approved the final manuscript.

Funding

Not applicable.

Availability of data and materials

All the data are available without restriction. Researchers can obtain data from the corresponding author.

Ethics approval and consent to participate

Not applicable.

Consent for publication

Not applicable.

\section{Competing interests}

The author(s) declare that they have no competing interests.

\section{Author details}

${ }^{1}$ Department of Thyroid and Breast Surgery, The First Affiliated Hospital of Chengdu Medical College, 278 Baoguang Avenue Middle Section, Xindu District, Chengdu City 610500, Sichuan Province, China. ${ }^{2}$ Department of Breast Surgery, Mianyang Central Hospital, Mianyang, Sichuan, China.

Received: 30 March 2020 Accepted: 4 June 2020

Published online: 15 June 2020

References

1. Anderson BO, Yip CH, Smith RA, Shyyan R, Sener SF, Eniu A, et al. Guideline implementation for breast healthcare in low-income and middle-income countries: overview of the breast health global initiative global summit 2007. Cancer. 2008;113(8 suppl):2221-43.

2. Bauer KR, Brown M, Cress RD, Parise CA, Caggiano V. Descriptive analysis of estrogen receptor (ER)-negative, progesterone receptor (PR)-negative, and HER2-negative invasive breast cancer, the so-called triple-negative phenotype. Cancer. 2007;109(9):1721-8.

3. Anders CK. Carey LA. Biology metastatic patterns and treatment of patients with triple-negative breast cancer. Clin Breast Cancer. 2009;9(suppl 2):S7381.

4. Reis-Filho JS, Tutt AN. Triple negative tumours: a critical review. Histopathology. 2008;52:108-18.

5. Jeruss JS, Mittendorf EA, Tucker SL, Gonzalez-Angulo AM, Buchholz TA, Sahin AA, et al. Combined use of clinical and pathologic staging variables to define outcomes for breast cancer patients treated with neoadjuvant therapy. J Clin Oncol. 2008;26(2):246-52

6. Kuerer HM, Newman LA, Smith TL, Ames FC, Hunt KK, Dhingra K, et al. Clinical course of breast cancer patients with complete pathological primary tumor and axillary lymph node response to doxorubicin-based neoadjuvant chemotherapy. J Clin Oncol. 1999;17(2):460-9. 
7. Liedtke C, Mazouni C, Hess KR, André F, Tordai A, Mejia JA, et al. Response to neoadjuvant therapy and long-term survival in patients with triplenegative breast cancer. J Clin Oncol. 2008;26(8):1275-81.

8. Huober J, von Minckwitz G, Denkert C, Tesch H, Weiss E, Zahm DM, et al. Effect of neoadjuvant anthracycline-taxane-based chemotherapy in different biological breast cancer phenotypes: overall results from the GeparTrio study. Breast Cancer Res Treat. 2010;124(1):133-40.

9. Carey LA, Dees EC, Sawyer L, Gatti L, Moore DT, Collichio F, et al. The triple negative paradox: primary tumor chemosensitivity of breast cancer subtypes. Clin Cancer Res. 2007;13(8):2329-34.

10. Rouzier R, Perou CM, Symmans WF, Ibrahim N, Cristofanilli M, Anderson K, et al. Breast cancer molecular subtypes respond differently to preoperative chemotherapy. Clin Cancer Res. 2005;11:5678-85.

11. Ring AE, Smith IE, Ashley S, Fulford LG, Lakhani SR. Lakhani Oestrogen receptor status, pathological complete response and prognosis in patients receiving neoadjuvant chemotherapy for early stage breast cancer. $\mathrm{Br} J$ Cancer. 2004;91:2012-7.

12. Clifton K, Gutierrez-Barrera A, Ma J, Bassett R, Litton J, Kuerer H, et al. Adjuvant versus neoadjuvant chemotherapy in triple-negative breast cancer patients with BRCA mutations. Breast Cancer Res Treat. 2018;170:101-9.

13. Fisher CS, Ma CX, William E, Aft RL, Eberlein TJ, Gao F, et al. Neoadjuvant chemotherapy is associated with improved survival compared with adjuvant chemotherapy in patients with triple-negative breast cancer only after complete pathologic response. Ann Surg Oncol. 2012;19:253-8.

14. Carlie R. Kennedy, Feng Gao, Julie a Margenthaler. Neoadjuvant versus adjuvant chemotherapy for triple negative breast cancer. J Surg Res. 2010; 163:52-7.

15. Sharma P, Kimler BF, Klemp JR, CS C. Outcomes with neoadjuvant versus adjuvant chemotherapy for T1-2 node negative triple negative breast cancer. J Clin Oncol. 2015;33:15.

16. Cheng YC, Smith E, Yen T. Abstract P5-16-09: overall survival of patients with non-metastatic triple negative breast cancer who received neoadjuvant vs adjuvant chemotherapy: cohort analysis of National Cancer Data Base (NCDB) 2010-2011. Cancer Res. 2017;77:4.

17. Yang H, Zhou L, Wang S, Cao Y, Tong F, Liu P, et al. Retrospective analysis of concurrent docetaxel and epirubicin neoadjuvant versus adjuvant chemotherapy: which leads to better outcomes for different subtype breast cancer patients? Medicine. 2018:97:40.

18. Biswas T, Jimmy T. Efird, Prasad S, Jindal C, Paul R. the survival benefit of neoadjuvant chemotherapy and $\mathrm{pCR}$ among patients with advanced stage triple negative breast cancer. Oncotarget. 2017;8(68):112712-9.

19. Bagegni NA, Tao Y, Ademuyiwa FO. Clinical outcomes with neoadjuvant versus adjuvant chemotherapy for triple negative breast cancer: a report from the National Cancer Database. PLoS One. 2019;14(9):e0222358.

20. Philipovskiy A, Corral J, Dwivedi KA, Heydarian R, Gaur S. Efficacy of neoadjuvant versus adjuvant chemotherapy in Hispanic/Latino $\mathrm{H} / \mathrm{L}$ women with local or locally advanced triple-negative breast cancer(TNBC). In Vivo. 2019;33(4):1227-34.

21. Stang A. Critical evaluation of the Newcastle-Ottawa scale for the assessment of the quality of nonrandomized studies in meta-analyses. Eur J Epidemiol. 2010;25(9):603-5.

22. Tierney JF, Stewart LA, Ghersi D, Burdett S, Sydes MR. Practical methods for incorporating summary time-to-event data into meta-analysis. Trials. 2007;8:16.

23. Egger $M$, Smith GD, Schneider $M$, et al. Bias in meta-analysis detected by a simple, graphical test. BMJ. 1997:315(7109):629-34.

24. Wolmark N, Wang J, Mamounas E, Bryant J, Fisher B. Preoperative chemotherapy in patients with operable breast cancer: nine-year results from national surgical adjuvant breast and bowel project B-18. Monogr Natl Cancer Inst. 2001:30:96-102.

25. van der Hage JA, van de Velde CJ, Julien JP, Tubiana-Hulin M, Vandervelden C, Duchateau L. Preoperative chemotherapy in primary operable breast cancer: results from the European Organization for Research and Treatment of cancer trial 10902. J Clin Oncol. 2001;19(22):4224-37.

26. Mauriac L, MacGrogan G, Avril A, Durand M, Floquet A, Debled M, et al. Neoadjuvant chemotherapy for operable breast carcinoma larger than $3 \mathrm{~cm}$ : a unicentre randomized trial with a 124-month median follow-up. Ann Oncol. 1999;10(1):47-52.

27. Lin NU, Claus E, Sohl J, Razzak AR, Arnaout A, Winer EP. Sites of distant recurrence and clinical outcomes in patients with metastatic triple-negative breast cancer. Cancer. 2008;113:2638.
28. Dawood S, Broglio K, Esteva FJ, Yang W, Kau SW, Islam R, et al. Survival among women with triple receptor-negative breast cancer and brain metastases. Ann Oncol. 2009;20:621.

29. Haque W, Verma V, Hatch S, Suzanne Klimberg V, Brian Butler E, Teh BS. Response rates and pathologic complete response by breast cancer molecular subtype following neoadjuvant chemotherapy. Breast Cancer Res Treat. 2018;170:559-67.

30. Early Breast Cancer Trialists' Collaborative Group (EBCTCG). Long-term outcomes for neoadjuvant versus adjuvant chemotherapy in early breast cancer: meta-analysis of individual patient data from ten randomised trials. Lancet Oncol. 2018;19:27-39.

31. Mauri D, Pavlidis N, loannidis JP. Neoadjuvant versus adjuvant systemic treatment in breast cancer: a meta-analysis. J Natl Cancer Inst. 2005:97(3):188-94.

32. Baron LF, Baron PL, Ackerman SJ, Durden DD, Pope TL Jr. Sonographically guided clip placement facilitates localization of breast cancer after neoadjuvant chemotherapy. AJR Am J Roentgenol. 2000;174:539-40.

33. von Minckwitz G, Untch M, Blohmer JU, Costa SD, Eidtmann H, Fasching PA et al. Definition and impact of pathologic complete response on prognosis after neoadjuvant chemotherapy in various intrinsic breast cancer subtypes. J Clin Oncol. 2012·30:1796-804

\section{Publisher's Note}

Springer Nature remains neutral with regard to jurisdictional claims in published maps and institutional affiliations.
Ready to submit your research? Choose BMC and benefit from:

- fast, convenient online submission

- thorough peer review by experienced researchers in your field

- rapid publication on acceptance

- support for research data, including large and complex data types

- gold Open Access which fosters wider collaboration and increased citations

- maximum visibility for your research: over $100 \mathrm{M}$ website views per year

At $\mathrm{BMC}$, research is always in progress.

Learn more biomedcentral.com/submissions 